\title{
Variations in Preference for Topical Vehicles Among Demographic Groups
}

\author{
Karen Figenshau, MD; Brooks David Kimmis, MD; Paul Reicherter, MD
}

\section{PRACTICE POINTS}

- Variations exist in preference for topical vehicles by age group, gender, and ethnicity.

- Identifying and utilizing preferred treatment options can help maximize patient outcomes.

Patient satisfaction and outcomes can be optimized by identifying preferences for topical vehicles by age, gender, and ethnicity. Our study sought to identify variations in preferences for creams, lotions, and ointments among different age groups, genders, and ethnicities. Each demographic group revealed statistically significant differences in vehicle preference $(P<.05)$.

Cutis. 2020;106:40-43

7 opical medication is a mainstay in the treatment of dermatologic conditions. Adherence to medication regimens can be challenging in patients requiring long-term topical treatment, and nonadherence is multifactorial. A major modifiable contributing factor is patient dissatisfaction with the vehicle used. Medications often have options for different topical preparations. Therefore, it is important to consider patient preference when prescribing topical treatments to maximize adherence, ensure patient satisfaction, and optimize outcomes.

We hypothesized that notable differences exist among demographic groups regarding preference for topical vehicles. Little research has been conducted to delineate trends. This study aimed to identify variations in preference for creams, lotions, and ointments by age, gender, and ethnicity.

\section{Methods}

Data were collected through surveys distributed to all patients seen at the Truman Medical Center University Health Dermatology Clinic in Kansas City, Missouri, between September 2018 and June 2019. The study was approved by the University of Missouri Kansas City institutional review board. An estimated response rate of 95\% was achieved. Each patient was informed that the survey was voluntary and anonymous, and declining to complete the survey had no effect on the care provided. Each patient completed only 1 survey and returned it to a collection box before departing from clinic.

In the survey, patients provided demographic information, including age, gender, and ethnicity. Age groups included patients younger than 40 years, 40 to 60 years, and older than 60 years. Gender groups included male and female. Ethnicity included white, black, Hispanic/ Latino, and Asian/Pacific Islander or other. Patients then chose 1 of 3 options for topical vehicle preference: cream, lotion, or ointment. Each of these options was accompanied by a brief description of the vehicle, a photograph, and examples of common commercial products to aid in decision-making. The expected values were calculated based on a probability distribution under the assumption that variables have no association. Therefore, the discrepancy between the expected value and the observed value

Dr. Figenshau is from the University of Missouri-Kansas City School of Medicine. Dr. Kimmis is from the University of Kansas School of Medicine, Kansas City. Dr. Reicherter is from Truman Medical Center University Health Dermatology Clinic, Kansas City, Missouri.

The authors report no conflict of interest.

Correspondence: Karen Figenshau, MD, University of Missouri-Kansas City School of Medicine, 2411 Holmes St, Kansas City, MO 64108

(kgf8v2@mail.umkc.edu). 
was used to describe the significance of the association between variables.

Data were analyzed using $\chi^{2}$ tests with the aid of a statistician. $P<.05$ was considered statistically significant.

\section{Results}

A total of 404 surveys were collected and recorded. Data showed statistically significant trends in each demographic parameter.

Age-First, we analyzed differences in preference based on age (Table 1). Of 404 patients, 163 were younger than 40 years, 171 were aged 40 to 60 years, and 70 were older than 60 years. Patients younger than 40 years preferred lotion (68 vs 46.0 expected). Patients aged 40 to 60 years showed preference for cream ( 83 vs 76.6 expected) and ointment (56 vs 46.1 expected). Patients older than 60 years preferred cream (41 vs 31.4 expected). These findings were statistically significant $(P<.0001)$.

Gender-Next, we evaluated variations based on gender (Table 2). Of 404 patients, 254 were female and 150 were male. Females preferred cream
(127 vs 113.8 expected). Males exhibited preference for lotion (50 vs 42.3 expected) and ointment (46 vs 40.5 expected). Differences between genders were statistically significant $(P=.023)$.

Ethnicity-We then analyzed preferences based on ethnicity (Table 3). Of 404 patients, 30 were Hispanic/ Latino, 26 were Asian/Pacific Islander or other, 227 were white, and 121 were black. Hispanic/Latino patients showed equivocal findings, aligning with expected counts. Asian/Pacific Islander or other patients exhibited slight preferences for cream (14 vs 11.6 expected) and lotion (10 vs 7.3 expected). White patients preferred cream (119 vs 101.7 expected) and lotion (82 vs 64.1 expected). Black patients showed strong preference for ointment (72 vs 32.6 expected). Differences in preferences based on ethnicity were statistically significant $(P<.0001)$.

\section{Comment}

Topical medication is a mainstay of dermatologic therapy. Many topical preparations (or vehicles) exist, including ointments, creams, lotions, gels, solutions, and

TABLE 1. Preference for Creams, Lotions, and Ointments Based on Age ( $\mathrm{N}=404)$

\begin{tabular}{|c|c|c|c|c|}
\hline \multirow[b]{2}{*}{ Age } & \multicolumn{3}{|c|}{ Patient Preference } & \multirow[b]{2}{*}{ Total } \\
\hline & Cream & Lotion & Ointment & \\
\hline \multicolumn{5}{|l|}{$<40 y$} \\
\hline Count & 57 & 68 & 38 & 163 \\
\hline Expected count & 73.0 & 46.0 & 44.0 & 163.0 \\
\hline \multicolumn{5}{|l|}{$40-60$ y } \\
\hline Count & 83 & 32 & 56 & 171 \\
\hline Expected count & 76.6 & 48.3 & 46.1 & 171.0 \\
\hline \multicolumn{5}{|l|}{$>60 y$} \\
\hline Count & 41 & 14 & 15 & 70 \\
\hline Expected count & 31.4 & 19.8 & 18.9 & 70.1 \\
\hline \multicolumn{5}{|l|}{ Total } \\
\hline Count & 181 & 114 & 109 & 404 \\
\hline Expected count & 181.0 & 114.1 & 109.0 & 404.1 \\
\hline
\end{tabular}

TABLE 2. Preference for Creams, Lotions, and Ointments Based on Gender $(\mathrm{N}=404)$

\begin{tabular}{lllll} 
& \multicolumn{3}{c}{ Patient Preference } & Total \\
\cline { 2 - 5 } Gender & Cream & Lotion & Ointment & 254 \\
\hline $\begin{array}{l}\text { Female } \\
\text { Count }\end{array}$ & 127 & 64 & 63 & 254.0 \\
$\begin{array}{c}\text { Expected count } \\
\text { Male }\end{array}$ & 113.8 & 71.7 & 68.5 & 150 \\
$\quad \begin{array}{l}\text { Count } \\
\text { Expected count }\end{array}$ & 54 & 50 & 46 & 150.0 \\
$\begin{array}{c}\text { Total } \\
\text { Count }\end{array}$ & 67.2 & 42.3 & 40.5 & 404 \\
\hline Expected count & 181 & 114 & 109 & 404.0 \\
\hline
\end{tabular}


TABLE 3. Preference for Creams, Lotions, and Ointments Based on Ethnicity ( $\mathrm{N}=404)$

\begin{tabular}{|c|c|c|c|c|}
\hline \multirow[b]{2}{*}{ Race } & \multicolumn{3}{|c|}{ Patient Preference } & \multirow[b]{2}{*}{ Total } \\
\hline & Cream & Lotion & Ointment & \\
\hline \multicolumn{5}{|l|}{ Hispanic/Latino } \\
\hline Count & 13 & 8 & 9 & 30 \\
\hline Expected count & 13.4 & 8.5 & 8.1 & 30.0 \\
\hline \multicolumn{5}{|c|}{ Asian/Pacific Islander or other } \\
\hline Count & 14 & 10 & 2 & 26 \\
\hline Expected count & 11.6 & 7.3 & 7.0 & 25.9 \\
\hline \multicolumn{5}{|l|}{ White } \\
\hline Count & 119 & 82 & 26 & 227 \\
\hline Expected count & 101.7 & 64.1 & 61.2 & 227.0 \\
\hline \multicolumn{5}{|l|}{ Black } \\
\hline Count & 35 & 14 & 72 & 121 \\
\hline Expected count & 54.2 & 34.1 & 32.6 & 120.9 \\
\hline \multicolumn{5}{|l|}{ Total } \\
\hline Count & 181 & 114 & 109 & 404 \\
\hline Expected count & 181.0 & 114.0 & 109.0 & 404.0 \\
\hline
\end{tabular}

foams. Vehicle type not only influences bioavailability of the prepared medication but also has a notable impact on adherence and subsequent efficacy of the topical therapy.

Medication adherence is especially challenging in dermatology, as topical medications play a central role in treatment. Compliance with the medication regimen is paramount in treatment efficacy. ${ }^{1}$ In dermatology, adherence with oral medications is higher than it is for topical medications ${ }^{2}$; various factors contribute to this difference. Compliance may decline with topical treatment due to time-consuming application, misunderstanding about the disease or the treatment regimen, frequency of administration, dissatisfaction with efficacy or appearance, and other variables. ${ }^{3}$

Other factors have been found to be important to topical medication adherence; younger age, female gender, marriage, employment, nonsmoking, nondrinking, and higher cognitive ability were associated with higher topical medication adherence. ${ }^{4}$ Our study focused on one factor: identification of demographic-specific preferences that might have implications on adherence within the studied demographic groups.

It is known that individual preferences exist when patients are choosing a topical preparation. However, a PubMed search of articles indexed for MEDLINE using the terms topical, vehicle, preparation, adherence, and preference revealed few studies that examined the preference for topical vehicle by age, gender, or ethnicity.

Existing studies have examined preferences for topical preparations based on specific disease states; this literature, albeit limited, demonstrates that preferences for topical product formulations vary among acne, atopic dermatitis, and plaque psoriasis patients. ${ }^{5}$ Other studies focus on specific patient populations or medications. For example, one study found that preference for corticosteroid vehicles among psoriasis patients was highly variable and choice of vehicle was critical to adherence. ${ }^{6}$ Another study highlighted differences in vehicle choice between younger and older age groups with psoriasis. ${ }^{7}$

Given the limited data overall, it was our goal to determine if any patterns of preference existed by age, gender, or ethnicity, regardless of disease state or indication for topical product. Importantly, over-the-counter products-cosmetic or otherwise-were not differentiated from prescribed topical medications. Our survey elucidated significant differences in preference by age, gender, and ethnicity.

Notable Findings-Regarding age, patients younger than 40 years preferred lotion, patients aged 40 to 60 years preferred cream, and patients older than 60 years preferred cream. Analysis based on gender showed that females preferred cream, and males preferred lotion and ointment. Analysis based on ethnicity most notably demonstrated a strong preference for ointment in black patients while showing preference for cream in white patients.

Potential Biases and Pitfalls_-Limitations of this study included the small Hispanic/Latino and Asian/Pacific Islander populations surveyed, possible misunderstanding of the survey by respondents, and the potential for surveys being filled out twice by the same patient. Future surveys could be conducted over a longer period to increase the total sample size and to better characterize less-represented populations, such as Hispanic and Asian patients. To avoid repeat participation, the first question of 
the survey asked patients to indicate if they had previously completed the survey and instructed patients who had to return the repeat survey to the front desk.

To limit other errors, our survey included concise accessible descriptions of each preparation along with clear representative photographs and examples of common brands. Still, it is possible that some mistakes could have been made while patients filled out the survey based on comprehension deficits, oversight, or other reasons. It also is possible that preference might vary individually depending on the indication of the topical productcosmetic or therapeutic - or even by anatomic site of application. Neither of these considerations was assessed specifically in our survey.

\section{Conclusion}

Our hope is that this study helps practitioners better anticipate topical preferences among patients with the ultimate goal of increasing medication adherence and patient outcomes. Nevertheless, although these general trends can provide helpful guidance, we acknowledge that individual preferences vary, and care should always be patient centered.
Acknowledgment-We thank An-Lin Cheng, PhD (Kansas City, Missouri), for assistance with the statistical analysis.

\section{REFERENCES}

1. Kircik LH.Vehicles always matter. J Drugs Dermatol. 2019;18:s99.

2. Furue M, Onozuka D, Takeuchi S, et al. Poor adherence to oral and topical medication in 3096 dermatological patients as assessed by the Morisky Medication Adherence Scale-8. Br J Dermatol. 2015;172:272-275.

3. Tan X, Feldman SR, Chang, J, et al. Topical drug delivery systems in dermatology: a review of patient adherence issues. Expert Opin Drug Deliv. 2012;9:1263-1271.

4. Ahn CS, Culp L, Huang WW, et al. Adherence in dermatology. J Dermatolog Treat. 2017;28:94-103.

5. Eastman WJ, Malahias S, Delconte J, et al. Assessing attributes of topical vehicles for the treatment of acne, atopic dermatitis, and plaque psoriasis. Cutis. 2014;94:46-53.

6. Felix K, Unrue E, Inyang M, et al. Patients preferences for different corticosteroid vehicles are highly variable. J Dermatolog Treat. 2019; 31:147-151.

7. Hong C-H, Papp KA, Lophaven KW, et al. Patients with psoriasis have different preferences for topical therapy, highlighting the importance of individualized treatment approaches: randomized phase IIIb PSO-INSIGHTFUL study. J Eur Acad Dermatol Venereol. 2017;31:1876-1883. 